\title{
Approximate solution for the two-dimensional Volterra-Integro differential equation of fractional order by using Generalized Deferential Transform method
}

\author{
Mohammed G. S. AL-Safi ${ }^{1 *}$, Wurood R. Abd AL- Hussein ${ }^{1}$, Muna S. Abbas ${ }^{1}$ \\ ${ }^{1}$ Department of Accounting, Al-Esraa University College, Baghdad, Iraq \\ *Corresponding author: mohammed.ghazi@esraa.edu.iq
}

Received 22/4/2018, Accepted 5/7/2018, published 23/7/2018.

DOI: $10.52113 / 2 / 05.02 .2018 / 1-9$

\begin{abstract}
In this work, an efficient generalized differential transform method (GDTM) is proposed for solving the twodimensional Volterra-Integro differential equation (2-DVIDE) of fractional order. The results of the proposed method are compared with exact solution, a numerical example is considered for testing the accuracy and validity of this method.
\end{abstract}

(C) 2018 Al Muthanna University. All rights reserved.

Keywords: Fractional calculus, Caputo derivative, Generalized differential transform method (GDTM).

The application of integral equations and integro

\section{Introduction}

The fractional calculus showed up in 1695 by Leibniz to L'Hospital. The subject of fractional calculus has picked up significance amid the previous three decades due for the most part to its showed applications in various areas of engineering and physics, which can be successfully described by mathematical models of fractional calculus [1-6]. Therefore, several numerical techniques are used to get the exact and the approximate solutions of these models, see for instance [7-10]. differential equations is an important manner within applied physics, they are appearing and considers as an important part in many numerous fields of social, biological, physical and engineering. $[11,12]$.

Zhou in 1986 [13] was proposed the differential transform method (DTM), the differential transform method has been created for tackling different sort of differential equations. Chen [14] has built up the differential transform method for solving partial differential. Ayaz [15] has applied DTM to differential-algebraic equations. 
Momani and Odibat they introduced the generalized differential transforms method (GDTM) [16-18].

There are different strategies for solving two-dimensional integral or integro-differential equations. Babolian [19] using Haar Wavelets for solving the nonlinear two-dimensional integral equations. Maleknejad [20] utilizing Block pulse functions to get solutions for nonlinear VolterraFredholm integral equations. Nemati [21] he used orthogonal polynomials for solving a class of two-dimensional nonlinear Volterra integral equations. Tari [22] he used the differential transform method to solve two-dimensional and nonlinear Volterra integral equations. In Mckee [23] utilize Euler algorithm for solving the twodimensional Volterra integral equations of the first kind.

Those association for whatever remains of this article is as follows. In section 2 we present a few important definitions of the fractional calculus theory and generalized differential transforms method. While in section 3 we illustrate how generalized differential transforms method may be used to solve 2-DVIDE of fractional order, also we give an numerical example to demonstrate the effectiveness of the present method, concluding remarks are given in the final section.

\section{Description of the method}

We start this section by given some fundamental definitions and properties of the fractional calculus theory and GDTM which utilized as a part of this paper.

\section{Definition (2.1) [10]}

The Riemann-Liouville fractional integral operator ${ }_{0} I_{x}^{v} f(x)$ of order $v>0$ is defined as follows:

$$
\begin{aligned}
& { }_{0} I_{x}^{v} f(x)=\frac{1}{\Gamma(v)} \int_{0}^{x}(x-t)^{v-1} f(t) d t, x>0 \\
& { }_{0} I_{x}^{0} f(x)=f(x)
\end{aligned}
$$

Where $\Gamma(v)$ is the Gamma function.

And satisfies the following properties for $\varepsilon>0$,

1. ${ }_{0} I_{x}^{v}\left({ }_{0} I_{x}^{\varepsilon}\right)={ }_{0} I_{x}^{v+\varepsilon}$,

2. ${ }_{0} I_{x}^{v} x^{\gamma}=\frac{\Gamma(\gamma+1)}{\Gamma(v+\gamma+1)} x^{v+\gamma} ; v \geq 0, \gamma>-1, t>0$.

\section{Definition (2.2) [10]}

The Caputo fractional derivative ${ }_{0}^{c} D_{x}^{v} f(x)$ of order $v>0$ is defined as:

$$
\begin{gathered}
{ }_{0}^{c} D_{x}^{v} f(x)= \\
\left\{\begin{array}{l}
\frac{1}{\Gamma(n-v)} \int_{0}^{x}(x-t)^{n-v-1} f^{(n)}(t) d t, \mathrm{n}-1<v<\mathrm{n} \\
\frac{d^{n}}{d x^{n}} f(x), \quad v=n \in \mathbb{N}
\end{array}\right.
\end{gathered}
$$

and satisfies the following properties:

1. ${ }_{0}^{c} D_{x}^{v} C=0, C$ constant;

2. ${ }_{0}^{c} D_{x}^{v} x^{\gamma}=\frac{\Gamma(\gamma+1)}{\Gamma(\gamma-v+1)} x^{\gamma-v}, x>0, \gamma>-1$.

\section{Definition (2.3) [10]}

The time-fractional derivative in the Caputo sense ${ }_{0}^{c} D_{t}^{v} u(x, t)$ for the function $u(x, t)$ of order $v>0$ is defined as: 


$$
\begin{aligned}
& { }_{0}^{c} D_{t}^{v} u(x, t)=\frac{\partial^{v} u(x, t)}{\partial t^{v}}= \\
& \left\{\begin{array}{l}
\frac{1}{\Gamma(n-v)} \int_{0}^{t}(t-\tau)^{n-v-1} \frac{\partial^{n} u(x, \tau)}{\partial \tau^{n}} d \tau, n-1<v<n \\
\frac{\partial^{n} u(x, t)}{\partial t^{n}}, \quad v=n \in \mathbb{N}
\end{array}\right.
\end{aligned}
$$

\section{Theorem (2.1) [16-18]}

If $U(k), F(k)$ and $G(k)$ are the differential transform (DT) of the functions $u(x), \mathrm{f}(x)$ and $\mathrm{g}(x)$ respectively then:

1. If $u(x)=f(x) \pm g(x)$ then

$$
U(k)=F(l) \pm G(l)
$$

2. If $u(x)=\alpha f(x)$ then

$$
U(l)=\alpha F(l)
$$

3. If $u(x)=f(x) g(x)$ then

$$
U(l)=\sum_{d=1}^{l} F(d) G(k-d)
$$

4. If $u(x)=\frac{d f(x)}{d x}$ then

$$
U(l)=(l+1) F(l+1)
$$

5. If $u(x)=\frac{d^{m} f(x)}{d x^{m}}$ then

$$
U(l)=(l+1)(l+2) \ldots(l+m) F(l+m)
$$

6. If $u(x)=\int_{0}^{x} f(t) d t$ then

$$
U(l)=\frac{F(l-1)}{l}, U(0)=0
$$

7. If $u(x)=x^{m}$ then

$$
U(l)=\delta(l-m)= \begin{cases}1, & l=m \\ 0, & 0 . W .\end{cases}
$$

8. If $u(x)=\sin (w x+v)$ then

$$
U(l)=\frac{w^{l}}{l !} \sin \left(\frac{l \pi}{2}+v\right)
$$

9. If $u(x)=\cos (w x+v)$ then

$$
U(l)=\frac{w^{l}}{l !} \cos \left(\frac{l \pi}{2}+v\right)
$$

10. If $u(x)=e^{\lambda x}$ then $\mathrm{U}(1)=\frac{\lambda^{l}}{l !}$

\section{Definition (2.4) [24]}

Consider the analytical function $u(x, t)$ of two variables, which is defined on $\mathrm{B} \subseteq \mathbb{R}^{2}$ Where $\mathrm{B}=[0, \mathrm{X}] \times[0, \mathrm{~T}]$ and $\left(\mathrm{x}_{0}, \mathrm{t}_{0}\right) \in \mathrm{B}$.

The two- dimensional differential transform (2DDT) of $u(x, t)$ is denoted by $U(1, s)$ and is defined on $\mathbb{N}^{2} \cup\{(0,0)\}$ as the following:

$\mathrm{U}(\mathrm{l}, \mathrm{s})=\left.\frac{1}{1 ! \mathrm{s} !}\left[\frac{\partial^{\mathrm{l}+\mathrm{s}} \mathrm{u}(\mathrm{x}, \mathrm{y})}{\partial \mathrm{x}^{\mathrm{l}} \partial \mathrm{y}^{\mathrm{s}}}\right]\right|_{\left(\mathrm{x}_{0}, t_{0}\right)}$

Where $u(x, t)$ is the original function. The inverse (2-DDT) of $\mathrm{U}(1, \mathrm{~s})$ is defined as:

$u_{0}(\mathrm{x}, \mathrm{t})=$

$\sum_{\mathrm{l}=0}^{\infty} \sum_{\mathrm{s}=0}^{\infty} \mathrm{U}(\mathrm{l}, \mathrm{s})\left(\mathrm{x}-\mathrm{x}_{0}\right)^{\mathrm{s}}\left(\mathrm{t}-\mathrm{t}_{0}\right)^{\mathrm{l}}$

Since $u(x, t)$ is an analytical function, it is clear that $\mathrm{u}(x, t)=u_{0}(x, t)$.

a combination of Eq.(4) and Eq. (5), with $\left(x_{0}, t_{0}\right)=(0,0)$, then the function $u(x, t)$ become :

$u(x, t)=\left.\sum_{l=0}^{\infty} \sum_{s=0}^{\infty} \frac{1}{l ! s !}\left[\frac{\partial^{l+s} u(x, t)}{\partial x^{l} \partial t^{s}}\right]\right|_{(0,0)} x^{l} t^{s}$.

\section{Theorem (2.2) [24]}

Suppose that $U(l, s), V(l, s)$ and $W(l, s)$ are the (DT) of the functions $u(x, t), v(x, t)$ and $w(x, t)$ respectively;

1. If $u(x, t)=v(x, t) \pm w(x, t)$, then $U(l, s)=$ $V(l, s) \pm W(l, s)$

2. If $u(x, t)=a v(x, t), \mathrm{a} \in \mathbb{R}$, then $U(l, s)=$ $a V(l, s)$

3. If $u(x, t)=v(x, t) w(x, t)$, then $U(l, s)=$ $\sum_{r=0}^{l} \sum_{p=0}^{s} V(r, s-p) W(l-r, p)$

4. If $u(x, t)=\left(x-x_{0}\right)^{l}\left(t-t_{0}\right)^{s}$, then $U(l, s)=\delta(l-n) \delta(s-m)$ 


\section{Theorem (2.3) [25]}

If $u(x, t)=f(x) g(t)$ and the function $f(x)=$ $x^{\mu} w(x)$, where $\mu>-1, w(x)$ has the Taylor series expansion of the form $w(x)=\sum_{f=0}^{\infty} c_{f}\left(x-x_{0}\right)^{v k}$, and

(a) $\varepsilon<\mu+1$ and $v$ is arbitrary, or

(b) $\varepsilon \geq \mu+1$ and $v$ is arbitrary and $c_{f}=0$

$$
\begin{aligned}
& \text { for } f=0,1,2, \ldots, q-1, \text { where } \\
& q-1<\varepsilon \leq q .
\end{aligned}
$$

Then the (GDT) of (5) becomes

$U_{v, \varepsilon}(l, s)=$

$\frac{1}{\Gamma(v \mathrm{l}+1) \Gamma(\varepsilon s+1)}\left[{ }_{0}^{c} D_{x_{0}}^{v l}\left({ }_{0}^{c} D_{t_{0}}^{\varepsilon}\right)^{S} u(x, t)\right]_{\left(x_{0}, t_{0}\right)}$

The generalized differential transform inverse of $\mathrm{U}_{v, \varepsilon}(\mathrm{l}, \mathrm{s})$ is given by

$\mathrm{u}(\mathrm{x}, \mathrm{t})=$

$\sum_{\mathrm{l}=0}^{\infty} \sum_{\mathrm{s}=0}^{\infty} \mathrm{U}_{v, \varepsilon}(\mathrm{l}, \mathrm{s})\left(\mathrm{x}-\mathrm{x}_{0}\right)^{\mathrm{sv}}\left(\mathrm{t}-\mathrm{t}_{0}\right)^{1 \varepsilon}$

\section{Theorem (2.4) [25]}

1- If $u(x, t)={ }_{0}^{c} D_{x_{0}}^{v} v(x, t), 0<v \leq 1$, then

$$
U_{v, \varepsilon}(l, s)=\frac{(v(l+1)+1)}{\Gamma(v l+1)} V_{v, \varepsilon}(l+1, s)
$$

2- If $v(x, t)=f(x) g(t)$, the function $f(x)$ satisfies the conditions given in theorem(2.2) and $u(x, t)={ }_{0}^{c} D_{x_{0}}^{\gamma} v(x, t)$, then

$$
U_{v, \varepsilon}(l, s)=\frac{\Gamma(v l+\gamma+1)}{\Gamma(v l+1)} V_{v, \varepsilon}\left(l+\frac{\gamma}{v}, s\right)
$$

\section{Theorem (2.5) [24]}

If $u(x, t)=\int_{0}^{x} \int_{0}^{y} f(s, t) d s d t, U(l, s)$ and $F(l, s)$ are the (DT) of the functions $u(x, t)$ and $f(x, y)$, respectively, then
$U(l, s)= \begin{cases}0, & \text { if } l=0 \text { or } s=0 ; \\ \frac{F(l-1, s-1)}{l s} & \text { if } l, s=1,2,3, \ldots\end{cases}$

\section{Theorem (2.6) [24]}

If $u(x, t)=\int_{0}^{x} \int_{0}^{y} f(s, t) g(s, t) d s d t, U(l, s)$, $F(l, s)$ and $G(l, s)$ are the (DT) of the functions $u(x, t), f(x, t)$ and $g(x, t)$, respectively, then $U(l, s)=$

$\left\{\begin{array}{c}0, \quad \text { if } l=0 \text { or } s=0 ; \\ \frac{1}{l s} \sum_{r=0}^{l-1} \sum_{p=0}^{s-1} F(r, s-p-1) G(l-r-1, s), \text { if } l, s=1,2,3, \ldots\end{array}\right.$

\section{Finding Approximate Solution}

Consider the (2-DVIDE) of fractional order of the form:

$\frac{\partial^{v} u(x, t)}{\partial x^{v}}+\frac{\partial u(x, t)}{\partial t}=-1+e^{x}+e^{t}+e^{x+t}+$

$\int_{0}^{x} \int_{0}^{t} u(s, y) d s d y$

Which have the exact solution $u(x, t)=e^{x+t}$. $x, t \in[0,1]$

with the initial conditions:

$\left\{\begin{array}{l}u(x, 0)=e^{x} \\ u(0, t)=e^{t}\end{array}\right.$

According to The (GDTM) present previously in Section 2, equation (12) can be written as follows:

$\frac{\Gamma(v(l+1)+1)}{\Gamma(v l+1)} U_{v, \varepsilon}(l+1, s)+(s+1) U(l, s+1)=$
$-\delta(l) \delta(s)+\frac{\delta(s)}{s !}+\frac{\delta(l)}{l !}+\frac{\delta(l) \delta(s)}{l ! s !}+\frac{U(l-1, s-1)}{l s}$

Then

$U_{v, \varepsilon}(l+1, s)=\frac{\Gamma(v l+1)}{\Gamma(v(l+1)+1)}[-(s+1) U(l, s+1)-$

$\delta(l) \delta(s)+\frac{\delta(l)}{l !}+\frac{\delta(s)}{s !}+\frac{\delta(l) \delta(s)}{l ! s !}+\frac{U(l-1, s-1)}{l s}$

The (GDTM) of the initial conditions (12) are given by; 
$\begin{cases}U_{v, \varepsilon}(l, 0)=\frac{1}{l !} & l=0,1,2, \ldots \\ U_{v, \varepsilon}(0, s)=\frac{1}{s !} & s=0,1,2, \ldots\end{cases}$

Now, if $1=0, s=1$ in (14):

$$
U_{v, \varepsilon}(1,1)=\frac{1}{\Gamma(v+1)}\left[-2 \frac{1}{2 !}+1\right]=0
$$

For $\mathrm{l}=0, \mathrm{~s}=2$

$U_{v, \varepsilon}(1,2)=\frac{1}{\Gamma(v+1)}\left[-3 \frac{1}{3 !}+\frac{1}{2}\right]=0$

For $1=0, s=3$

$U_{v, \varepsilon}(1,3)=\frac{1}{\Gamma(v+1)}\left[-4 \frac{1}{4 !}+\frac{1}{6}\right]=0$

$U_{v, \varepsilon}(1, s)=0$,

Also, if $1=1, s=1$ in (14):

$U_{v, \varepsilon}(2,1)=\frac{\Gamma(v+1)}{\Gamma(2 v+1)}[-2(0)+1]=\frac{\Gamma(v+1)}{\Gamma(2 v+1)}$

For $1=1, s=2$

$U_{v, \varepsilon}(2,2)=\frac{\Gamma(v+1)}{\Gamma(2 v+1)}\left[-3(0)+\frac{1}{2}\right]=\frac{1}{2} \frac{\Gamma(v+1)}{\Gamma(2 v+1)}$

For $1=1, s=3$

$U_{v, \varepsilon}(2,3)=\frac{1}{3 !} \frac{\Gamma(v+1)}{\Gamma(2 v+1)}$

$U_{v, \varepsilon}(2,4)=\frac{1}{4 !} \frac{\Gamma(v+1)}{\Gamma(2 v+1)}$

Now, if $1=2, s=1$ in (14):

$U_{v, \varepsilon}(3,1)=\frac{\Gamma(2 v+1)}{\Gamma(3 v+1)}\left[-\frac{\Gamma(v+1)}{\Gamma(2 v+1)}+\frac{1}{2}\right]$

For $1=2, s=2$

$U_{v, \varepsilon}(3,2)=-\frac{\Gamma(v+1)}{2 ! \Gamma(3 v+1)}$

For $\mathrm{l}=2, \mathrm{~s}=3$

$U_{v, \varepsilon}(3,3)=-\frac{\Gamma(v+1)}{3 ! \Gamma(3 v+1)}$

$U_{v, \varepsilon}(3,4)=-\frac{\Gamma(v+1)}{4 ! \Gamma(3 v+1)}$
Similarly for $1=3, s=1$ in (14):

$U_{v, \varepsilon}(4,1)=\frac{\Gamma(3 v+1)}{\Gamma(4 v+1)}\left[\frac{\Gamma(v+1)}{\Gamma(3 v+1)}+\frac{1}{6}\right]$

Therefore, from (5), the approximate solution of the 2-DVIDE of fractional order for $v=\varepsilon=1$ and for different value of $\mathrm{m}$ and $\mathrm{n}$ can be given as:

$$
\begin{aligned}
& u_{3,3}(x, t)=\left(1+t+\frac{t^{2}}{2}+\frac{t^{3}}{3 !}\right)\left(x+\frac{x^{2}}{2}+\frac{t x^{2}}{2}+\right. \\
& \left.\frac{t^{2} x^{2}}{4} \ldots\right) \\
& u_{4,4}(x, t)=\left(1+t+\frac{t^{2}}{2 !}+\frac{t^{3}}{3 !}+\frac{t^{4}}{4 !}+x+\frac{x^{2}}{2 !}+\frac{x^{4}}{4 !}+\right. \\
& \left.\frac{t x^{2}}{2}+\frac{t x^{4}}{12}+\frac{t^{2} x^{2}}{4}-\frac{t^{2} x^{3}}{12}+\frac{t^{2} x^{4}}{48}+\frac{t^{3} x^{2}}{12}+\frac{t^{3} x^{4}}{144}\right) \\
& u_{8,8}(x, t)=\left(1+t+\frac{t^{2}}{2 !}+\frac{t^{3}}{3 !}+\frac{t^{4}}{4 !}+\frac{t^{5}}{5 !}+\frac{t^{6}}{6 !}+\frac{t^{6}}{6 !}+\right. \\
& \frac{t^{7}}{7 !}+\frac{t^{8}}{8 !}+x+\frac{x^{2}}{2 !}+\frac{x^{4}}{4 !}-\frac{x^{5}}{5 !}+\frac{x^{6}}{6 !}-\frac{x^{7}}{7 !}+\frac{x^{8}}{8 !}+\frac{t x^{2}}{2}+ \\
& \frac{t x^{4}}{12}-\frac{t x^{5}}{60}+\frac{t x^{6}}{240}-\frac{t x^{7}}{480}+\frac{t x^{8}}{4032}+\frac{t^{2} x^{2}}{4}-\frac{t^{2} x^{3}}{12}+\frac{t^{2} x^{4}}{4 !}- \\
& \frac{t^{2} x^{5}}{5 !}+\frac{t^{2} x^{6}}{288}-\frac{t^{2} x^{7}}{1440}+\frac{t^{2} x^{8}}{8960}+\frac{t^{3} x^{2}}{12}-\frac{t^{3} x^{3}}{36}+\frac{t^{3} x^{4}}{72}- \\
& \frac{t^{3} x^{5}}{240}+\frac{t^{3} x^{6}}{864}-\frac{t^{3} x^{7}}{5040}+\frac{t^{3} x^{8}}{20160}+\frac{t^{4} x^{2}}{48}-\frac{t^{4} x^{3}}{144}+\frac{t^{4} x^{4}}{288}- \\
& \left.\frac{t^{4} x^{5}}{960}+\frac{t^{4} x^{6}}{4320}-\frac{t^{4} x^{7}}{17280}+\cdots .\right)
\end{aligned}
$$

Following Table 1 represents the absolute error between the exact and approximate solution of the proposed method for Example 1 using different values of $n$ and $m$ when $v=\varepsilon=1$. Also Figures (1), (2) and (3) presents the comparison graphically between the results obtained by the exact and the approximate solution for various values of $\mathrm{x}$ and $\mathrm{t}$ with $n=$ $m=4,8,16$ when $v=\varepsilon=1$ 
Fig.(2):Represent the comparison between Exact and

Table1: Absolute error between the exact and the approximate solutions by GDTM for different values of $\mathrm{x}$ and $\mathrm{t}$.

\begin{tabular}{|c|c|c|c|c|}
\hline \multicolumn{2}{|c|}{} & n=m=4 & n=m=8 & n=m=16 \\
\hline$x$ & $t$ & $\begin{array}{c}\text { Absolute } \\
\text { error }\end{array}$ & $\begin{array}{c}\text { Absolute } \\
\text { error }\end{array}$ & $\begin{array}{c}\text { Absolute } \\
\text { error }\end{array}$ \\
\hline 0.25 & 0.25 & $7.44156304 \mathrm{e}-02$ & $7.44149467 \mathrm{e}-02$ & $7.44149468 \mathrm{e}-02$ \\
\hline 0.25 & 0.5 & $1.69157948 \mathrm{e}-01$ & $1.69425659 \mathrm{e}-01$ & $1.69425721 \mathrm{e}-01$ \\
\hline 0.5 & 0.25 & $1.67106489 \mathrm{e}-01$ & $1.66786755 \mathrm{e}-01$ & $1.66786748 \mathrm{e}-01$ \\
\hline 0.5 & 0.5 & $3.61131221 \mathrm{e}-01$ & $3.61084578 \mathrm{e}-01$ & $3.61084655 \mathrm{e}-01$ \\
\hline 0.75 & 0.25 & $2.88130207 \mathrm{e}-01$ & $2.85579904 \mathrm{e}-01$ & $2.85579640 \mathrm{e}-01$ \\
\hline 0.75 & 0.5 & $6.11334982 \mathrm{e}-01$ & $6.08665286 \mathrm{e}-01$ & $6.08665082 \mathrm{e}-01$ \\
\hline 1.0 & 0.25 & $4.49381586 \mathrm{e}-01$ & $4.38301569 \mathrm{e}-01$ & $4.38298029 \mathrm{e}-01$ \\
\hline 1.0 & 0.5 & $9.40890459 \mathrm{e}-01$ & $9.28058919 \mathrm{e}-01$ & $9.28054760 \mathrm{e}-01$ \\
\hline
\end{tabular}

Fig.(1):Represent the comparison between Exact and Approximation solution for above example using GDTM when $\mathrm{N}=\mathrm{M}=4, v=\varepsilon=1$.
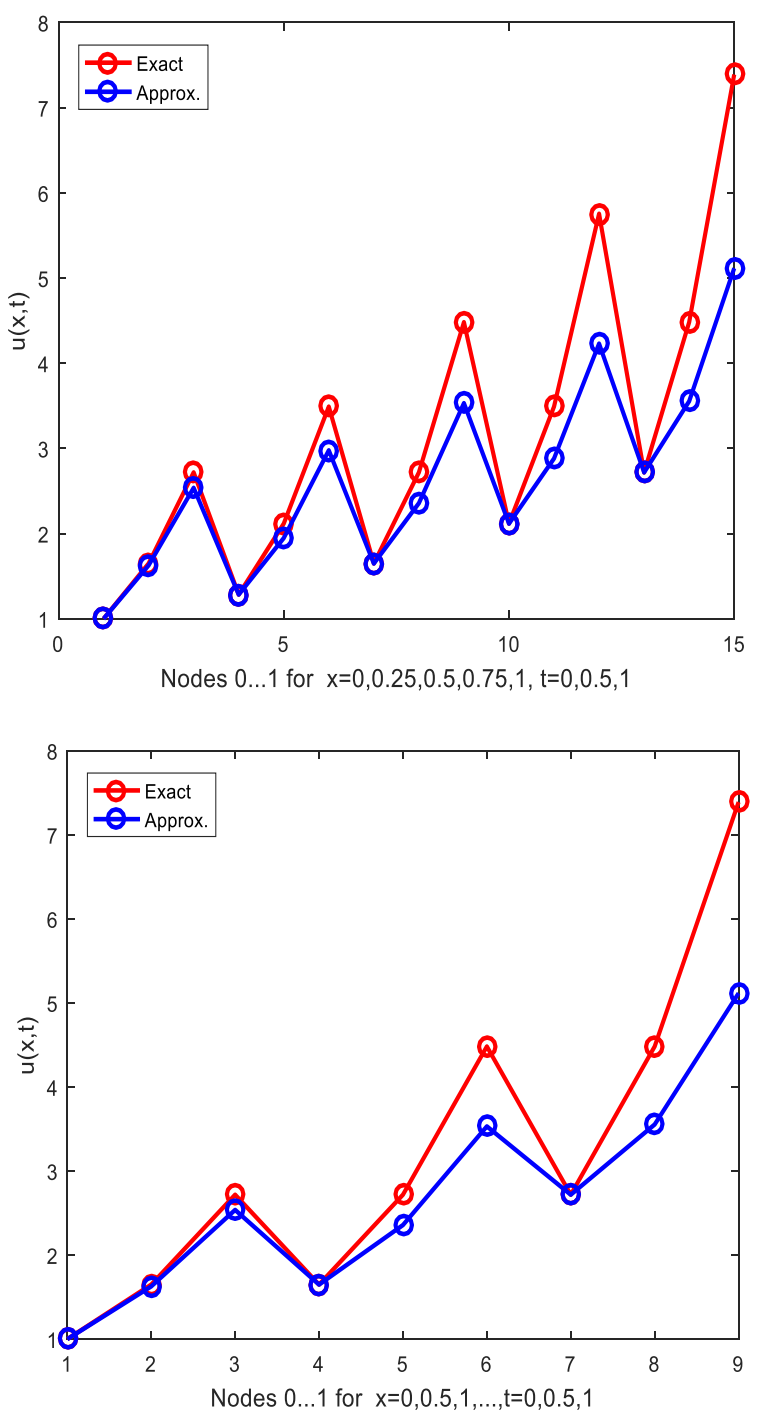

Approximation solution for above example using GDTM when $\mathrm{N}=\mathrm{M}=8, v=\varepsilon=1$. 


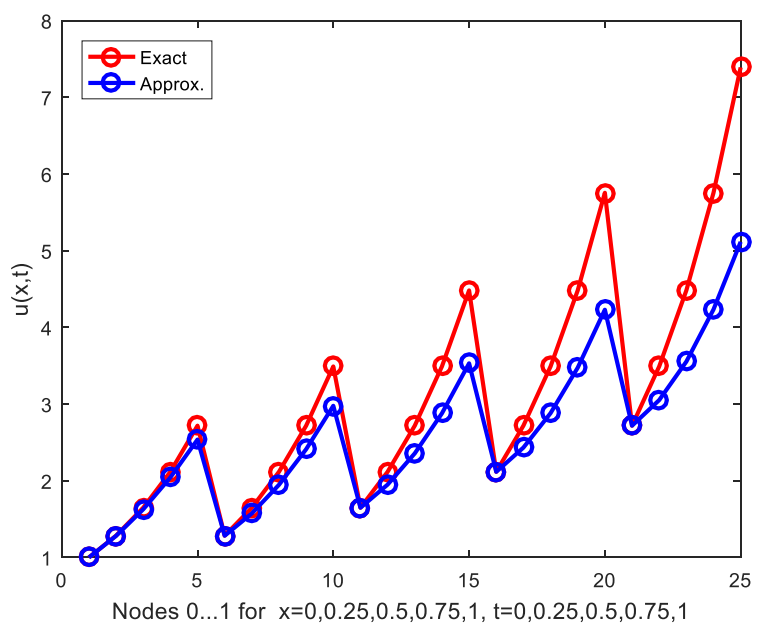

Fig.(3):Represent the comparison between Exact and Approximation solution for above example using GDTM when $\mathrm{N}=\mathrm{M}=16 . v=\varepsilon=1$.

\section{Conclusion}

In this article, the GDTM is used to get an approximate solution of the 2-DVIDE of fractional order. The fractional derivatives are given here in the Caputo sense because the Caputo fractional derivative allows traditional initial and boundary conditions to be included in the formulation of the problem. Comparisons are made between approximate solutions and exact solutions, the numerical result are obtained using Matlab R2016a to test the applicability and validity of the proposed algorithm.

\section{References}

[1] Osama H.M., Fadhel S.F., Mohammed G.S. AL-Safi., 2015, Sinc-Jacobi Collocation Algorithm For Solving The Time-Fractional
Diffusion-Wave Equations, international Journal of Mathematics and Statistics Studies UK.; Vol. 3, No. 1,pp. 28-37.

[2] Osama H.M., Fadhel S.F., Mohammed G.S. AL-Safi., 2015, Numerical solution for the time - Fractional Diffusion-wave Equations by using Sinc-Legendre Collocation Method, Mathematical Theory and Modeling, Vol. 5, No. 1,pp. 49-57.

[3] Osama H.M., Fadhel S.F., Mohammed G.S. AL-Safi., 2015, Shifted Jacobi tau method for solving the space fractional diffusion equations", IOSR Journal of Mathematics (IOSR-JM), No. 10, No. 3,pp. 34-44.

[4] Mohammed G.S AL-Safi, Farah L.J., Muna S.A., 2016, Numerical Solution for Telegraph Equation of Space Fractional Order using Legendre Wavelets Spectral tau Algorithm, Australian Journal of Basic and Applied Sciences, Vol. 10, No.12, pp. 383-391.

[5] Mohammed G.S. AL-Safi, Liqaa Z.H., 2017, Approximate Solution for advection dispersion equation of time Fractional order by using the Chebyshev waveletsGalerkin Method, Iraqi Journal of Science.; Vol. 58, No.3B, pp.1493-1502.

[6] Osama H.M, Mohammed G.S .AL-Safi, Ahmed A.Y. 2018, Numerical Solution for Fractional Order Space-Time Burger's Equation Using Legendre Wavelet - 
Chebyshev Wavelet Spectral Collocation Method, Journal of Al-Nahrain University, Vol. 21, No. 1, pp.121-127.

[7] Odibat Z., Momani S., 2006, Application of variational iteration method to nonlinear differential equations of fractional order, Int. J. Nonlinear Sci.Numer. Simul.Vol. 7, No. 1, pp.15-27.

[8] Momani S., Odibat Z., 2006 ,Analytical solution of a time-fractional Navier-Stokes equation by Adomian decomposition method, Appl. Math. Comput., Vol. 177 ,pp.488-494.

[9] Odibat Z., Momani S., 2006, Modified homotopy perturbation method: Application to quadratic Riccati differential equation of fractional order, Chaos Solitons $\quad$ Fractals (doi:10.1016/j.chaos.2006.06.041).

[10] Podlubny I., 1999, Fractional Differential Equations, Academic Press, New York.

[11] Kythe P. K. and Puri P., 1992, Computational methods for linear integral equations. University of New Orleans, New Orleans.

[12] Rashed M. T., 2004, Numerical solution of functional differential, integral and integrodifferential equations. Applied Numerical Mathematics, Vol. 156, pp. 485-492.

[13] Zhou J. K., 1986,Differential transform and its application for electric circuits.
Huazhong, University Press, Wuhan, China.

[14] Chen C. K., 1999,Solving partial differential equations by two-dimensional differential transform. Appl. Math. Comput., Vol. 106, pp. 171-179.

[15] Ayaz F., 2004,Application of differential transform method to differential-algebraic equations. Appl. Math. Comput., Vol. 152, pp. 649-657.

[16] Odibat Z., Shaher M. and Ertürk V.S., 2008, Generalized differential transform method: application to differential equations for fractional order.Appl. Math. Comput., Vol. 197, pp. 467-477.

[17] Momani S., Odibat Z, and Erturk V. S., 2007, Generalized differential transform method for solving a space and time fractional diffusion-wave equation,Physics Letters. A, vol. 370, no. 5-6, pp. 379-387.

[18] Odibat Z. and Momani S.,2008, A generalized differential transform method for linear partial differential equations of fractional order, Applied Mathematics Letters, vol. 21, no. 2, pp. 194-199.

[19] Babolian E., Bazm S. and Lima P., 2011,Numerical solution of nonlinear twodimensional integral equations rational Haar functions. Communications in nonlinear Science and Numerical Simulation, Vol. 16, No. 3, pp. 1164-1175. 
[20] Maleknejd K. and Mahdiani K., 2011,Solving nonlinear mixed Volterra Fredholm integral equations with twodimensional Block-pulse functions using direct method. Communications in Nonlinear Science and Numerical Simulation, Vol. 16, No. 9, pp. 3512-3519. [21] Nemate S.M, Lima PM. And Ordokhani Y., 2013, Numerical solution of a class of twodimensional nonlinear Volterra integral equations using Legendre polynomials. Journal of Computational and Applied Mathematics, Vol. 242, pp. 53-69.

[22] Tari A. Shahmorad S., Rahimi M.Y. and Talati F., 2009, Solving a class of twodimensional linear and nonlinear Volterra integral equations by the differential transform method. Journal of Computational and Applied Mathematics, Vol. 228, No. 1, pp. 70-76.
[23] Mckee S., Tang T. and Diogo T., 2000,An Euler-type method for two-dimentional Volterra integral equations of the first kind. IMA Journal of Numerical Analysis, Vol. 20 ,No. 2, pp. 423-440.

[24] Mohseni M. and Saeed H., 2010,Application of differential transforms for solving the Volterra integro-partial differential equations.Iranian Journal of Science and Technology, Transaction A, Vol. 34, No. A1, pp. 59-70.

[25] Cetinkaya A., Kymaz O. and Caml J. 2011, Solutions of Nonlinear PDE's of fractional Order with Generalized Differential Transform Method. International Mathematical Forum, Vol. 6, no.1, pp.3947. 